\title{
Hurricane Imaging Radiometer Wind Speed and Rain Rate Retrievals during the 2010 GRIP Flight Experiment
}

\author{
Saleem Sahawneh, IEEE Student Member, Spencer \\ Farrar, IEEE Student Member, James Johnson, \\ IEEE Senior Member, and W. Linwood Jones, IEEE \\ Life Fellow \\ Central FL Remote Sensing Lab \\ Department of EECS \\ University of Central Florida \\ Orlando, FL
}

\author{
Jason Roberts, Sayak Biswas, IEEE Member, \\ Daniel Cecil \\ NASA/Marshall Space Flight Center \\ Huntsville, AL
}

\begin{abstract}
Microwave remote sensing observations of hurricanes, from NOAA and USAF hurricane surveillance aircraft, provide vital data for hurricane research and operations, for forecasting the intensity and track of tropical storms. The current operational standard for hurricane wind speed and rain rate measurements is the Stepped Frequency Microwave Radiometer (SFMR), which is a nadir viewing passive microwave airborne remote sensor [1].

The Hurricane Imaging Radiometer, HIRAD, will extend the nadir viewing SFMR capability to provide wide swath images of wind speed and rain rate, while flying on a high altitude aircraft. HIRAD was first flown in the Genesis and Rapid Intensification Processes, GRIP, NASA hurricane field experiment in 2010. This paper reports on geophysical retrieval results and provides hurricane images from GRIP flights. An overview of the HIRAD instrument and the radiative transfer theory based, wind speed/rain rate retrieval algorithm is included. Results are presented for hurricane wind speed and rain rate for Earl and Karl, with comparison to collocated SFMR retrievals and WP3D Fuselage Radar images for validation purposes.
\end{abstract}

Keywords-synthetic aperture radiometry; SFMR; Microwave radiometry; Brightness Temperature; HIRAD

\section{INTRODUCTION}

The Hurricane Imaging Radiometer was developed by NASA, Marshall Space Flight Center, NOAA's Hurricane Research Division, the University of Michigan and the University of Central Florida. It measures surface emissivity and path average rain intensity over a wide swath of approximately 3 times the flying altitude, as shown in Fig. 1. HIRAD is a fourfrequency, C-band, synthetic aperture radiometer, with a $0.8 \mathrm{~m} \times$ $0.6 \mathrm{~m}, 37 \times 16$ element stacked patch array antenna [2]. The array is thinned to 10 active 16 element linear array antennas, enabling real aperture imaging along track and synthesis crosstrack over
36 baselines [3]. This provides a swath of approximately $\pm 60^{\circ}$ and a spatial resolution of approximately $2 \mathrm{Km}$ at nadir and $5 \mathrm{Km}$ at the swath edge. HIRAD flew on the NASA Johnson Space Center's WB-57 aircraft at $18 \mathrm{~km}$ altitude, over Hurricanes Earl and Karl, during GRIP, on September 1-2, 2010 and September 16, 2010, respectively. Simultaneously, NOAA, Hurricane Research Division flights with SFMR provided under-flight passes across the HIRAD swath, that enabled comparison and validation of the HIRAD measurements.

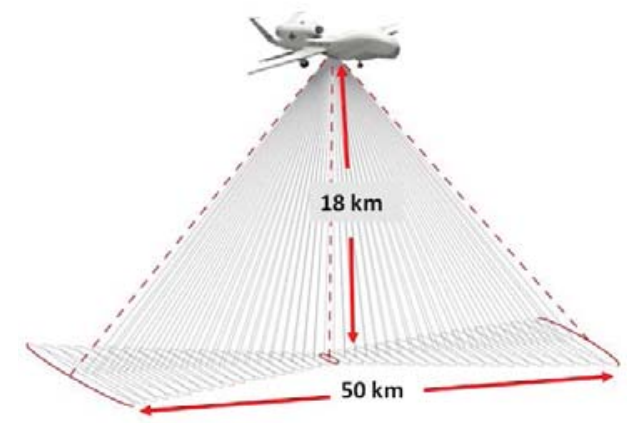

Fig. 1. HIRAD measurement geometry showing synthesized cross-track footprints.

Two geophysical retrieval methods have been applied to the HIRAD brightness temperature $T_{b}$ data, by the Central Florida Remote Sensing Lab (CFRSL). One uses single frequency empirical wind speed and rain rate algorithms, for near real-time applications [4]; and the other uses a rigorous, radiative transfer theory based method (maximum likelihood estimation, MLE). The single frequency approach, defines major features in an image, but isn't capable of clearing rain from the wind speed retrievals. The multi-frequency MLE method is fully capable of producing wind speed in the presence of rain, but is sensitive to 
$\mathrm{T}_{\mathrm{b}}$ calibration stability at all frequencies. The MLE retrievals are emphasized in this paper.

\section{GEOPHYSICAL RETRIEVAL METHOD}

A maximum likelihood estimation algorithm, based on a HIRAD Radiative Transfer Model (RTM), that includes a CFRSL surface emissivity model $[5,6]$ and the SFMR rain model, was developed by CFRSL. The surface emissivity model was designed to cover a wind speed range of $10-85 \mathrm{~m} / \mathrm{sec}$, and the rain model covers a range of approximately $5-100 \mathrm{~mm} / \mathrm{hr}$.

The Radiative Transfer Model is used to create tables of modeled brightness temperatures vs. all combinations of wind speed (WS) and rain rate (RR) in hurricanes, at each HIRAD frequency, for each measurement cell in the HIRAD swath. Both polarizations are included, to account for the cross-pol $T_{b}$ contribution in the outer swath. Tables exist for each $0.1 \mathrm{deg}$. in earth incidence angle (EIA), allowing precise interpolation for each measurement cell, and reducing the MLE process to iterations using look-up tables.

The MLE process uses the look-up tables and minimizes the total squared differences, between the observed and modeled brightness temperatures, over HIRAD frequencies. The combination of WS and RR producing the minimum squared difference yields the retrieved values.

\section{FLIGHT PATTERNS}

Both of the 2010 flights in Hurricanes Earl and Karl were at approximately $18 \mathrm{~km}$ in altitude on the WB-57, where Earl was in the Atlantic off the coast of southern Florida and Karl was in the Gulf of Mexico near the Yucatan Peninsula. A "box" pattern was flown in Earl, where successive legs at 90 deg. angles form a box. In Karl, a crisscrossing pattern was used, to better capture the center of the storm. A total of eight flight legs were flown in Earl over five hours, and 10 flight legs in Karl over seven hours.

The SFMR, on the NOAA P-3 aircraft, provided near simultaneous estimates of brightness temperature, wind speed, and rain rate in both Earl and Karl. SFMR flies at approximately 10,000 feet in a "Figure-4" pattern, which is centered on the hurricane eye, and includes legs that cross at the storm center. In Earl, SFMR flew directly across the HIRAD swath on the 4 primary legs (legs 1, 3, 4, and 6) that formed the box pattern, and captured the eye-wall features of the storm. In Karl, both instruments were flown in patterns passing over the storm center, so the SFMR underpasses transected the HIRAD swath more diagonally. Nevertheless, collocation comparisons covering the entire HIRAD swath were possible for both flights. SFMR wind speed and rain rate were useful as surface truth for the HIRAD measurements on both flights.

\section{HURRICANE EARL RETRIEVALS}

It was possible to identify storm centric collocations, between HIRAD and SFMR, within approximately 30 minutes of each other in Earl. These were used to validate the RTM and MLE retrieval algorithm. Results for WS comparisons on legs 3 and 4 are used as examples, to demonstrate the quality of the HIRAD retrievals. The MLE algorithm was also used to provide full WS and $\mathrm{RR}$ images for legs 1, 3, 4 and 6. A rain image, in $\mathrm{dBz}$, from the P3 Fuselage Radar was used, along with the SFMR RR retrievals, to validate HIRAD RR retrievals. Earl retrievals were done using 4, 5 and $6.6 \mathrm{GHz} \mathrm{T}_{\mathrm{b}}$ data only.

\section{A. HIRAD and SFMR Collocation Comparisons}

The NOAA P-3 flew 3 legs in Earl, to make a Figure-4, with SFMR crossing the storm center twice and completely traversing the HIRAD swath on all 4 legs. Fig. 2 shows the SFMR flight path overlayed on HIRAD legs 3 and 4, with the first leg of SFMR going from east to west through the storm center and crossing HIRAD leg 3, and the third leg going from south to north and crossing HIRAD leg 4.

The comparison between the SFMR WS and the HIRAD WS using the MLE for HIRAD legs 3 and 4 is shown in Fig. 3 and Fig. 4, respectively. The time difference between the SFMR pass and the collocated HIRAD measurements for leg 3 was approximately 7 minutes, and the difference was approximately 33 minutes for leg 4. The positive angles in Fig. 3 indicate the right hand side of the HIRAD swath, with respect to the flight direction, and the negative angles are the left hand side of the swath. Fairly good agreement exists between HIRAD and SFMR, particularly in the region of the eye-wall in the outer right hand swath. The better agreement across the full swath is on leg 3 , where the collocations are more time coincident. These collocation results show, that better agreement is probably achievable with improved $\mathrm{T}_{\mathrm{b}}$ calibration across the swath.

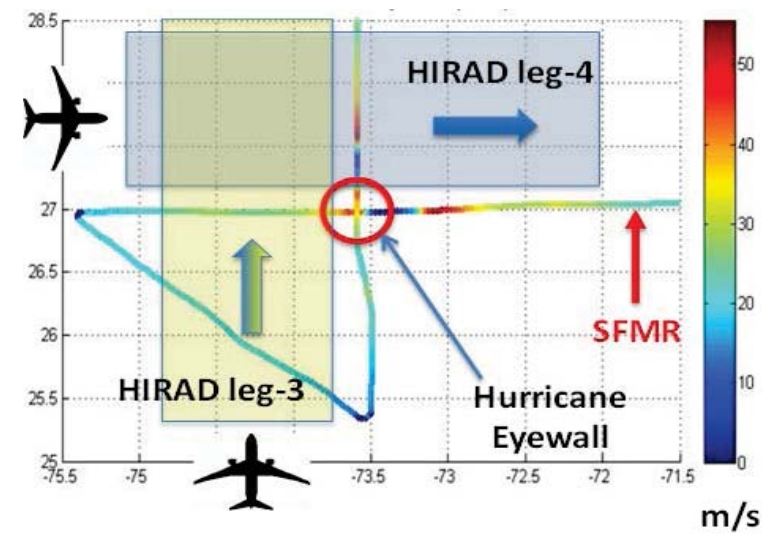

Fig. 2. SFMR transecting HIRAD legs $3 \& 4$ in Earl

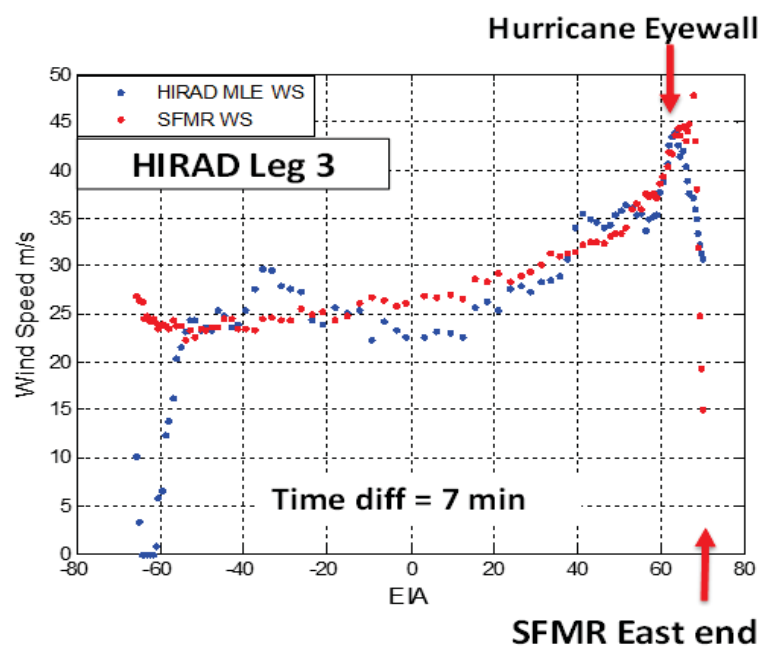

Fig. 3. SFMR transecting HIRAD legs 3 \& 4 in Earl 


\section{B. Retrieved WS and RR Images}

Fig. 5 and Fig. 6 show composite images made from individual images of legs $1,3,4 \& 6$. In these composites, retrieved values are averaged in the regions where the individual legs overlap. The swath in these images is $-50 \mathrm{deg}$. to $+60 \mathrm{deg}$. The eyewall region has good structure in these images and the maximum WS, and RR values were in good agreement with SFMR. Fig. 7 shows a RR image for leg 1 of the NOAA P3 Fuselage Radar. A secondary eye-wall, seen south of the storm center in the radar image, is also visible in the HIRAD rain image. Even though these initial images show good sensitivity and accuracy, even in the outer swath, they also show that improvement is needed in the $T_{b}$ calibration across the swath.

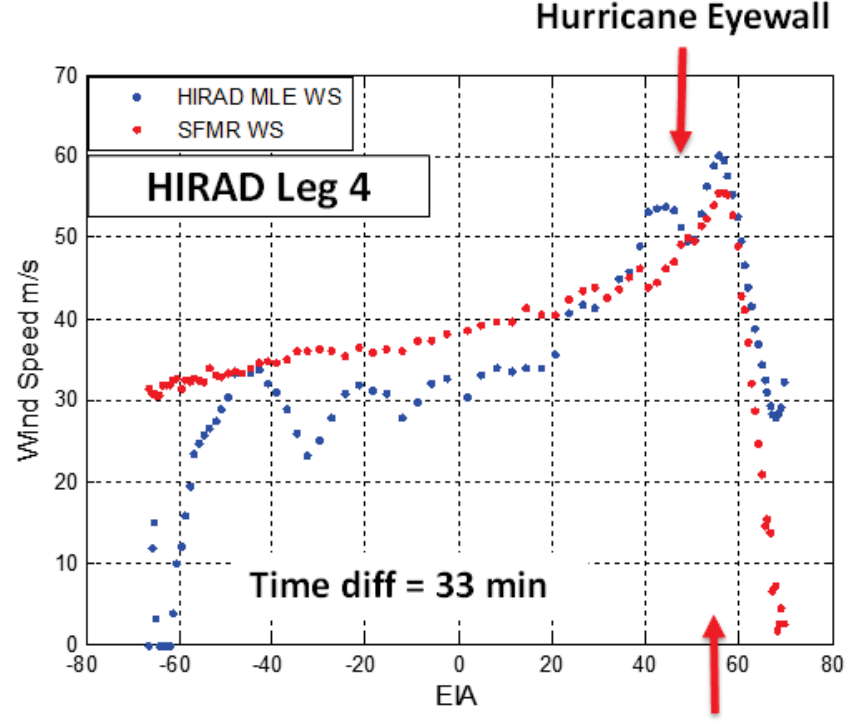

SFMR South end

Fig. 4. Collocated HIRAD leg 4 MLE and SFMR WS

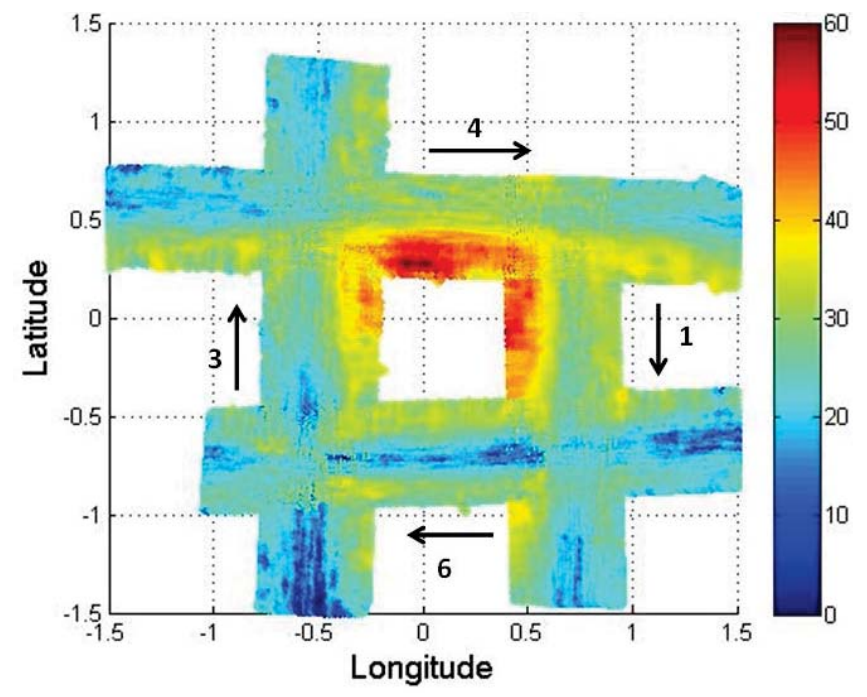

Fig. 5. Hurricane Earl Wind Speed $(\mathrm{m} / \mathrm{s})$ Composite Image plotted in storm centric coordinates

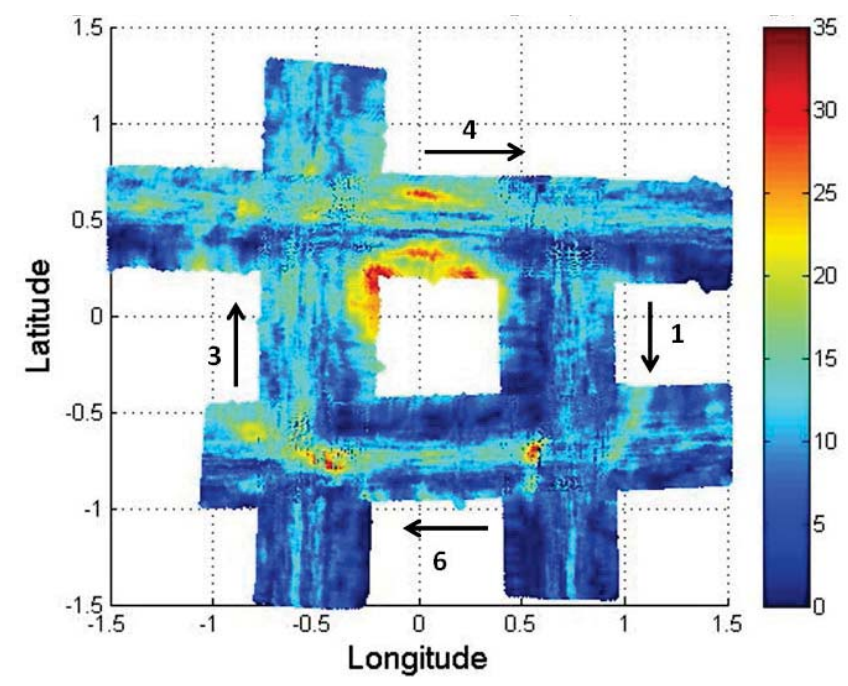

Fig. 6. Hurricane Earl Rain Rate ( $\mathrm{mm} / \mathrm{hr}$ ) Composite Image plotted in storm centric coordinates

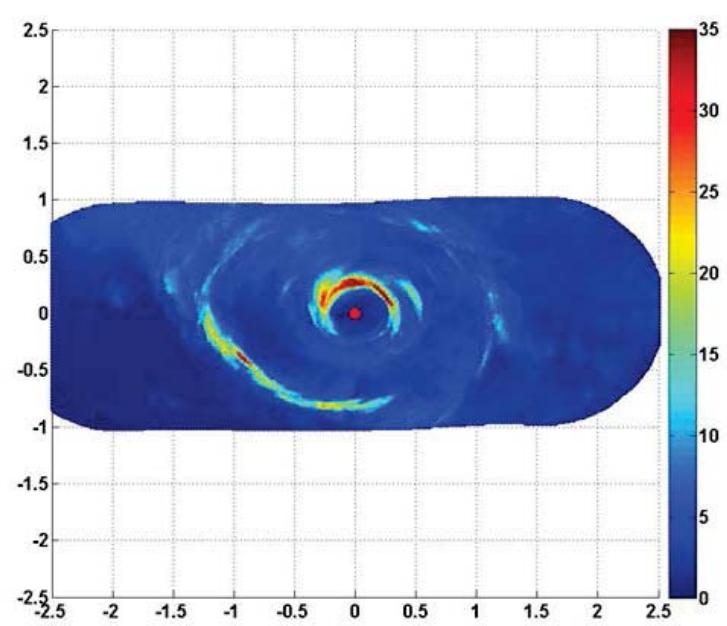

Fig. 7. Rain Rate (mm/hr) image for leg1 of the NOAA P3 Fuselage Radar. The red dot designates the storm center.

\section{V.HURRICANE KARL RETRIEVALS}

The HIRAD flight legs for Karl formed a crisscrossing pattern with the crossings at the storm center, so that legs 1,3 , 5,7 and 10 all crossed approximately at the eye. The NOAA P3 flew 2 figure-4 patterns providing SFMR data for surface truth on 3 passes through the storm. HIRAD retrievals used the 4,5 and $6.6 \mathrm{GHz} \mathrm{T}_{\mathrm{b}}$ data for Karl as well as Earl.

Hurricane Karl had a smaller eye and eye-wall structure than Earl and HIRAD demonstrated that, from this altitude it could map the entire inner-core surface wind and rain structure, for a hurricane of this size in a single pass. Composite images from legs 1 and 3 and from legs 7 and 10 are shown in Fig. 6 and Fig.7 for Karl.

The MLE algorithm was applied using three frequencies (4, $5,6.6 \mathrm{GHz}$ ) for the surface wind speed retrievals shown in Fig. 8 . This is the leg 1 and 3 example with the eye and eye-wall structure well defined. The estimated winds in the eye are biased high for a couple of reasons. First, cross-track calibration issues are present in Karl $\mathrm{T}_{\mathrm{b}}$ 's, similar to Earl. Plus, as the eye is imaged 
in the outer swath and the slant path passes through rain in the eye-wall, the additional attenuation will elevate the observed $T_{b}$. Retrieved WS values are averaged in the overlap regions of legs 1 and 3 here also. The magnitude of the wind in the eye-wall at $35-40 \mathrm{~m} / \mathrm{sec}$. agrees well with the SFMR peak wind observations.

Two and three frequency retrievals were computed, and the two frequency RR results provided more distinct features, shown in Fig. 9 and Fig 10. Better crosstrack calibration of the $6.6 \mathrm{GHz}$ $\mathrm{T}_{\mathrm{b}}$ 's should improve the 3 frequency retrievals. For example, the crosstrack variation patterns, in areas away from the storm center, are not as prevalent in Fig. 9 as in Fig. 8, where the 6.6 $\mathrm{GHz}$ signal influences the results. The agreement with SFMR is good for both images in Fig. 9, where the maximum RR values are $40-45 \mathrm{~mm} / \mathrm{hr}$. and the SFMR observed rain intensity up to approximately $50 \mathrm{~mm} / \mathrm{hr}$. on one particular pass.

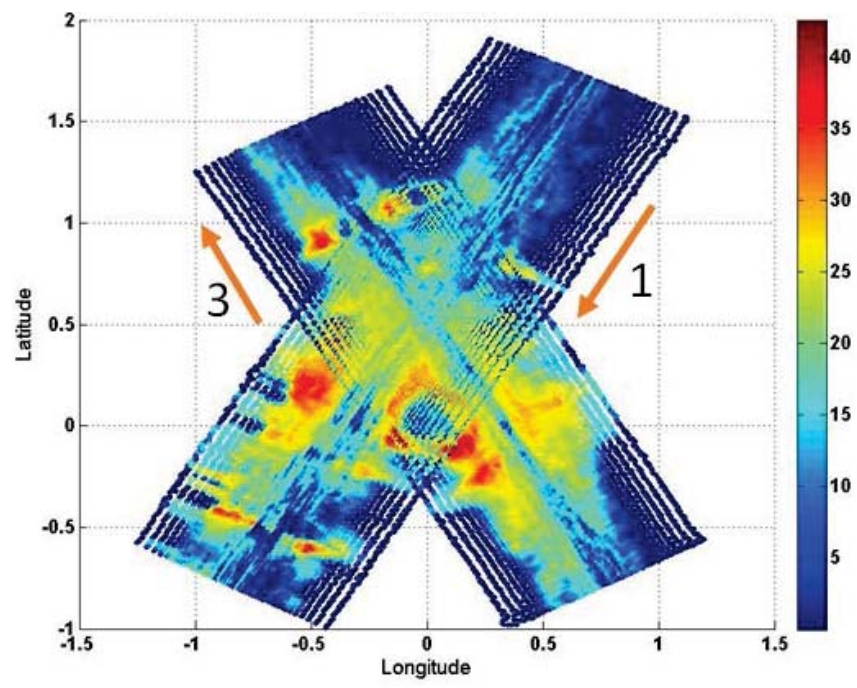

Fig. 8. Hurricane Karl Wind Speed (m/s) Composite Image for legs 1 \& 3 plotted in storm centric coordinate.

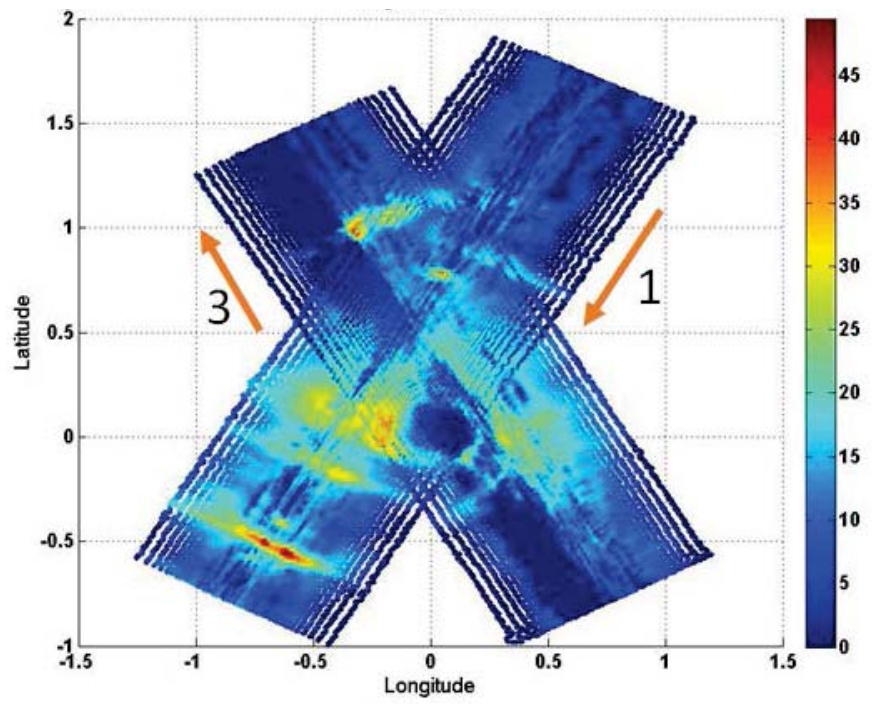

Fig. 9 Hurricane Karl Rain Rate (mm/hr) Composite Image for legs 1 \& 3 plotted in storm centric coordinate.

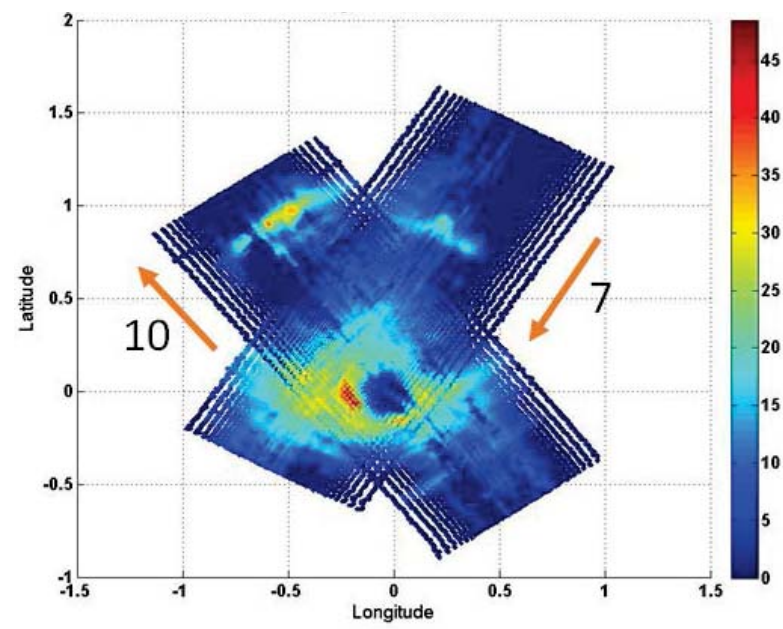

Fig. 10 composite image of Karl rain rate $(\mathrm{mm} / \mathrm{hr})$ for legs $7 \& 10$ plotted in storm centric coordinate.

CFRSL is currently developing improved calibration methods for HIRAD $\mathrm{T}_{\mathrm{b}}$ data, with emphasis on Karl, in order to get better wind speed and rain rate retrievals. Radiative transfer modeling and available surface truth, such as SFMR, P-3 radar, and H-Wind mapping products, are being used to provide final corrections to the flight data, prior to retrievals. Swath location dependent $\mathrm{T}_{\mathrm{b}}$ bias corrections, at each frequency, can be defined for each flight leg, using data from the beginning and end of each leg, where winds are relatively uniform and conditions are relatively rain free.

Fig. 11 shows a time series of $\mathrm{T}_{\mathrm{b}}$ at 4,5 and $6.6 \mathrm{GHz}$ and incidence angle 40 deg., on Karl leg 10. Each plot has been normalized (corrected) by its own unique function, that was determined by comparing the observed $\mathrm{T}_{\mathrm{b}}$ 's to modeled $\mathrm{T}_{\mathrm{b}}$ 's, at the beginning of leg 10 . The same normalization was applied to the entire leg, at each frequency. Fig. 8 shows good behavior over most of the leg. Variations in WS are visible and frequency independent, and rain bands are visible where frequency dispersion exists. Brightness temperature is higher with higher frequency over most of the leg, as it should be.

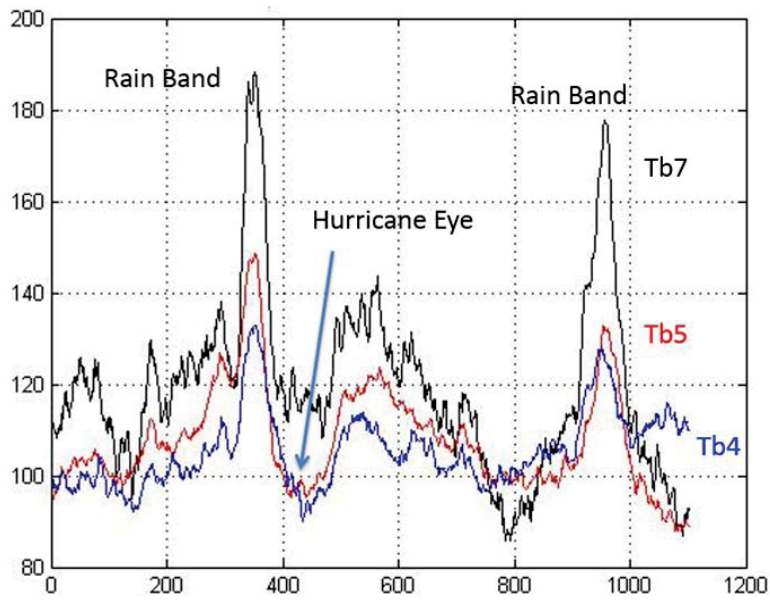

Fig. 11. Time series of normalized TB at incidence angle $40 \mathrm{deg}$. 


\section{CONCLUSION}

The HIRAD concept, with its broad swath measurement capability, offers the potential for significant improvement over the current SFMR. Using high-flying, long duration, unmanned aircraft such as the Global Hawk, real time tropical cyclone surveillance can be possible. The swath of HIRAD has been demonstrated at $50-60 \mathrm{~km}$.

HIRAD has demonstrated the ability to produce a wind speed and rain rate image in a single pass. Images from the 2010 GRIP Experiment, hurricanes Earl and Karl, demonstrated the ability to image the wind and rain structure in the eye and eye-wall of hurricanes. Comparison to SFMR and P-3 radar measurements showed good agreement in the magnitude of wind and rain intensity in the eye-wall, and the ability to identify large rain bands and small rain cells outside the storm center.

Under the HS3 program, hardware and calibration methods have been implemented that will improve accuracy and stability. Future HIRAD retrievals will improve with better calibrated $\mathrm{T}_{\mathrm{b}}$ 's.

\section{REFERENCES}

[1] Uhlhorn, E. W., P. G. Black, J. L. Franklin, M.Goodberlet, J. Carswell and A. S. Goldstein, "Hurricanesurface wind measurements from an operational steppedfrequencymicrowave radiometer," Mon. Wea. Rev ., 135 (9):3070-3085, 2007.

[2] M. C. Bailey, Ruba A. Amarin, et.al., "Multi-Frequency Synthetic Thinned Array Antenna for the Hurricane Imaging Radiometer" IEEE Transactions on Antennas and Propogation, Vol. 58, No. 8, pp. 2562-2570, August 2010.

[3] C. S. Ruf, "Numerical annealing of low redundancy linear arrays," IEEE Trans. Antennas Propag., vol. 41, no. 1, pp. 85-90, 1993.

[4] Timothy L. Miller, et.al., "The Hurricane Imaging Radiometer: Present and Future" Proc. IGARSS 2013, Melbourne, 21-26 July 2013.

[5] Salem F. El-Nimri, "An Improved C-Band Ocean Surface Emissivity Model at Hurricane-Force Wind Speeds Over a Wide Range of Earth Incidence Angles" IEEE GEOSCIENCE AND REMOTE SENSING LETTERS, VOL. 7, NO. 4, pp. 641 - 645, OCTOBER 2010.

[6] Ruba A. Amarin; Jones, W. L.; El-Nimri, S.F.; Johnson, J. W.; Ruf, C. S.; Miller, T. L. and E. Uhlhorn; "Hurricane Wind Speed Measurements in Rainy Conditions using the Airborne Hurricane Imaging Radiometer (HIRAD)"; Trans. GeoSci. Remote Sens., vol. 50, issue: 1, pp. 180-192, Jan. 2012. 